\title{
Formation and destabilization of Ga interstitials in GaAsN: Experiment and theory
}

\author{
P. Laukkanen, ${ }^{1}$ M. P. J. Punkkinen, ${ }^{1,2,{ }^{*}}$ J. Puustinen, ${ }^{3}$ H. Levämäki ${ }^{1}$ M. Tuominen, ${ }^{1}$ K. Schulte,,${ }^{4}$ J. Dahl,${ }^{1}$ J. Lång, ${ }^{1}$ \\ H. L. Zhang, ${ }^{2}$ M. Kuzmin, ${ }^{5}$ K. Palotas, ${ }^{6}$ B. Johansson, ${ }^{2,7}$ L. Vitos, ${ }^{2,7,8}$ M. Guina, ${ }^{3}$ and K. Kokko ${ }^{1}$ \\ ${ }^{1}$ Department of Physics and Astronomy, University of Turku, FI-20014 Turku, Finland \\ ${ }^{2}$ Applied Materials Physics, Department of Materials Science and Engineering, Royal Institute of Technology, SE-10044 Stockholm, Sweden \\ ${ }^{3}$ Optoelectronics Research Centre, Tampere University of Technology, FI-33101 Tampere, Finland \\ ${ }^{4}$ MAX-lab, Lund University, SE-221 00 Lund, Sweden \\ ${ }^{5}$ Ioffe Physical-Technical Institute of the Russian Academy of Sciences, St. Petersburg 194021, Russian Federation \\ ${ }^{6}$ Department of Theoretical Physics, Institute of Physics, Budapest University of Technology and Economics, \\ Budafoki út. 8, H-1111 Budapest, Hungary \\ ${ }^{7}$ Department of Physics and Astronomy, Division of Materials Theory, Uppsala University, Box 516, SE-751210 Uppsala, Sweden \\ ${ }^{8}$ Research Institute for Solid State Physics and Optics, Wigner Research Center for Physics, P.O. Box 49, H-1525 Budapest, Hungary
}

(Received 5 June 2012; revised manuscript received 5 September 2012; published 9 November 2012)

\begin{abstract}
Using first-principles total energy calculations we have found complex defects induced by $\mathrm{N}$ incorporation in GaAsN. The formation energy of the Ga interstitial atom is very significantly decreased due to local effects within the defect complex. The stability of the Ga interstitials is further increased at surfaces. The present results suggest that the energetically favorable $\mathrm{Ga}$ interstitial atoms are much more abundant in GaAsN than the previously considered $\mathrm{N}$ defects, which have relatively large formation energies. Our synchrotron radiation core-level photoemission measurements support the computational results. The formation of harmful Ga interstitials should be reduced by incorporating large group IV B atoms in GaAsN.
\end{abstract}

DOI: 10.1103/PhysRevB.86.195205

PACS number(s): 61.72.jj, 68.35.Dv, 71.55.Eq, 81.05.Ea

\section{INTRODUCTION}

$\mathrm{Ga}(\mathrm{In}) \mathrm{AsN}$ dilute nitrides $(<5 \% \mathrm{~N})$ have attracted significant interest due to their unique properties and opportunities they render for development of optoelectronic devices. ${ }^{1-9}$ For example, dilute nitride enables epitaxy of heterostructures with a $1 \mathrm{eV}$ band gap that are lattice matched to GaAs, thus offering a very attractive avenue for increasing the efficiency of multijunctions. ${ }^{5}$ The development of dilute nitride solar cells is particularly important as the enabler of future concentrated photovoltaic systems requiring cells with conversion efficiencies approaching $50 \%$. As far as the epitaxy is concerned, dilute nitride technology has progressed a long way towards fulfilling its exceptional promises. ${ }^{10}$ However, fundamental studies linking the material synthesis to material properties are still required to elucidate the intriguing mechanisms of defect formation and $\mathrm{N}$ incorporation in novel heterostructures. In particular for solar cells, the mitigation of defects is critical as the defects are limiting the maximum current generated by the cells.

Various harmful crystal defects are formed and trapped in the grown films, in addition to the desired substitution of As by $\mathrm{N}\left(\mathrm{N}_{\mathrm{As}}\right)$. It is known that even a small amount of $\mathrm{N}$ incorporated into $\mathrm{Ga}(\mathrm{In})$ As deteriorates the crystalline quality, decreasing the luminescence intensity and carrier lifetime of $\mathrm{Ga}(\mathrm{In}) \mathrm{AsN}$ films. ${ }^{1,11,12}$ The post-growth heating of $\mathrm{Ga}(\mathrm{In}) \mathrm{AsN}$ films is known to improve the luminescence to some extent, ${ }^{1,12,13}$ indicating removal of defects, but also to shift the emission peak to shorter wavelengths (a phenomenon called blueshift). A significant number of studies have focused on the amount and nature of $\mathrm{N}$-induced defects in the complex $\mathrm{Ga}(\mathrm{In}) \mathrm{AsN}$ system, ${ }^{14-25}$ because these defects (nonsubstitutional $\mathrm{N}$ atoms or related defects) are considered as the main source for the reduced luminescence intensity and carrier lifetime.
Yet, the results are incomplete and partly conflicting. There are experiments which show only a negligible amount of N-induced interstitials, ${ }^{19}$ while some other experimental results reveal a much more significant amount of interstitial atoms. ${ }^{20,24}$ On the other hand, calculations ${ }^{14,17,25}$ have shown that the $(\mathrm{N}-\mathrm{N})_{\text {As }}$ split interstitial (i.e., N-N dimer substitutes $\mathrm{As}),(\mathrm{N}-\mathrm{As})_{\mathrm{As}}$ split interstitial, $\mathrm{As}_{\mathrm{Ga}}-\mathrm{N}_{\mathrm{As}}$ complex (i.e., As antisite plus $\mathrm{N}_{\mathrm{As}}$ ), and $\mathrm{N} t^{\mathrm{Ga}}$ interstitial (i.e., $\mathrm{N}$ in the center of tetrahedron formed by nearest neighbor $\mathrm{Ga}$ atoms) are energetically favored among various defect models. However, the amount of these defects should be negligible under normal growth conditions, because the defects become energetically favored compared to substitutional $\mathrm{N}$ only if the position of the Fermi level is close to the conduction band corresponding to heavy $n$ doping. ${ }^{17,25}$ Recent experiments ${ }^{22,23}$ revealed Ga interstitial defects, which is very surprising due to their high formation energy. Moreover, these Ga interstitials were shown to form a nonradiative recombination channel. Blocking of the nonradiative recombination channel through the Ga interstitial atoms was shown to increase the photoluminescence intensity by a factor of 8 , which is a direct proof of the detrimental effect of this type of defects. ${ }^{23}$ Under similar growth conditions these defects have never been seen in $\mathrm{N}$-free GaAs. ${ }^{23}$

In this work we show that the formation energy of a Ga interstitial atom is decreased significantly by local bonding effects induced by surrounding $\mathrm{N}$ atoms. Furthermore, we show that surface effects also decrease the formation energy of these Ga interstitials. In order to probe bonding sites in GaAsN, we have utilized high-resolution core-level photoelectron spectroscopy in combination with $a b$ initio core-level shifts calculated for various $\mathrm{N}$ bonding environments. This chemically sensitive approach provides information complementary to the previous characterizations. 


\section{CALCULATIONAL METHODS}

Calculations were performed using an $a b$ initio density functional theory total-energy method within the local density approximation (LDA). ${ }^{26,27}$ The approach is based on the plane wave basis and projector augmented wave method ${ }^{28,29}$ (Vienna ab initio simulation package, VASP) ${ }^{30-33}$ The optimization of the atomic structure was performed using conjugate-gradient minimization of the total energy with respect to the atomic coordinates. Atoms were relaxed until the remaining forces were less than $20 \mathrm{meV} / \AA$. All our bulk calculations were performed using a cubic 512-atom supercell consisting of $4 a \times 4 a \times 4 a 8$-atom GaAs unit cells ( $a=5.63 \AA$ ). The plane wave cutoff energy of $400 \mathrm{eV}$ was used. Ga $3 d$ electrons were treated as core electrons. The $k$-point sampling was carried out using the Monkhorst-Pack scheme ${ }^{34}$ with the origin shifted to the $\Gamma$ point by a $2 \times 2 \times 2$ mesh.

The formation energy $E_{f}$ of a $\mathrm{Ga}$ interstitial atom in the charge state $q$ is defined as

$$
\begin{aligned}
E_{f}\left[\mathrm{Ga}_{I}^{q}\right]= & E_{\mathrm{tot}}\left[\mathrm{Ga}_{I}^{q}\right]-E_{\mathrm{tot}}[\mathrm{Ref}]-\mu_{\mathrm{Ga}} \\
& +q\left[E_{F}+E_{v}+\Delta V\right],
\end{aligned}
$$

where $E_{\text {tot }}\left[\mathrm{Ga}_{\mathrm{I}}^{q}\right]$ is the total energy of a supercell containing a $\mathrm{Ga}$ interstitial atom, while $E_{\text {tot }}[\mathrm{Ref}]$ is the total energy of the corresponding supercell without the interstitial atom (reference system). As a reference system for the Ga interstitial we use bulk GaAs. For GaAsN, the reference system is bulk GaAs with $\mathrm{N}$ substitutional atoms (on As sites) as distant from each other as possible. The chemical potential for $\mathrm{Ga}$ $\left(\mu_{\mathrm{Ga}}\right)$ is obtained using an orthorhombic bulk cell. ${ }^{35}$ A lower limit is obtained for the formation energy corresponding to Ga-rich conditions. The Fermi energy $\left(E_{F}\right)$ is given with respect to the top of the valence band. For charged cases, a neutralizing background is introduced to the supercell. The chemical potential of a removed electron $\left\{q\left[E_{F}+E_{v}+\Delta V\right]\right\}$ depends on the position of the Fermi level, and thus, on doping. The energy of an electron at the top of the valence band $\left(E_{\mathrm{v}}\right)$ is obtained by ${ }^{36}$

$$
E_{v}=E_{\text {tot,bulk }}^{q=0}-E_{\text {tot,bulk }}^{q=+1}
$$

where $E_{\text {tot,bulk }}$ is the total energy of the bulk supercell. Due to the well known problems of the LDA to reproduce experimental band gaps, the position of the Fermi level is allowed to vary in the energy range determined by the experimental band gap of $1.52 \mathrm{eV} .^{37}$ Through the potential correction $\Delta V$ the valence band maxima of different systems are aligned. This is estimated by calculating the average electrostatic potential at the ion cores. The average electrostatic potential at an ion core far from the defect is compared to the corresponding atom in the bulk supercell. ${ }^{25,38}$ The same kind of procedure is used to estimate the initial state core-level shifts. The average electrostatic potentials at the defect $\mathrm{N}$ atoms are compared to Ga (or As) potentials far from the defect. This way the $\mathrm{N}$ core-level shifts can be determined as well.

For the surface calculations we use $\mathrm{a}(2 \times 1)$ structure within an $(8 \times 8)$ surface slab cell with four $\mathrm{N}$ atoms at positions of the model structure with and without the Ga interstitial atom. The $k$ mesh is $2 \times 2 \times 1$.

\section{EXPERIMENTS}

Before the GaAsN growth, a 160-nm GaAs buffer layer was grown on $n$-type GaAs(100) wafer pieces by molecular beam epitaxy (MBE) at $580{ }^{\circ} \mathrm{C}$. After that the substrate temperature was decreased to $475^{\circ} \mathrm{C}$ when a 20-nm-thick GaAsN layer was grown with an $\mathrm{As} / \mathrm{Ga}$ beam equivalent pressure ratio of $18, \mathrm{~N}$ flow of $0.25 \mathrm{sccm}$, and with $450 \mathrm{~W}$ plasma power resulting in an $\mathrm{N}$ content of $3.30 \%$. An amorphous As cap layer was deposited on the GaAsN surface, by decreasing the substrate temperature slowly from $475^{\circ} \mathrm{C}$ to room temperature under the As flux in $\mathrm{MBE}$, to protect it against oxidation and contamination during the sample transfer via air into a photoemission chamber.

After loading the sample into the photoemission chamber, it was annealed in ultrahigh vacuum at about $400{ }^{\circ} \mathrm{C}$. That removed the protective As cap including various As oxides and provided the GaAsN films free of surface contaminants. Low-energy electron diffraction from this surface showed a $(2 \times 4)$ pattern. It can be expected that this sample heated at $400{ }^{\circ} \mathrm{C}$ represents largely the as-grown GaAsN (grown at $\left.470{ }^{\circ} \mathrm{C}\right)$.

Photoemission measurements were performed with the synchrotron-radiation facilities at the MAX-lab (Sweden) on the beamline I311. In the spectral analysis, the Shirley background removal was used. Each spectrum was fitted with the Voigt-profile peaks. The minimum number of components, as deduced from pure line shapes (i.e., peak shoulders, asymmetries, and broadening) was included in the fittings.

Photoluminescence (PL) spectra were measured from separate GaAsN quantum-well samples (grown with the same molecular beam epitaxy equipment as the samples used in photoemission measurements) including three 20-nm GaAsN quantum wells and 50-nm GaAs barriers.

\section{RESULTS AND DISCUSSION}

Figure 1(a) [1(b)] represents the calculated formation energies for $\mathrm{Ga}$ interstitial atoms in the center of the tetrahedron formed by four As (Ga) atoms under Ga-rich conditions. The results are in qualitative agreement with those of Zhang and Northrup ${ }^{39}$ and Malouin et al. ${ }^{40}$ One can note that the formation energies are positive and quite large, and that they depend on the charge state of the interstitial atom.

The corresponding formation energies for the considered model structure including four nitrogen atoms as shown in Fig. 2(a) are represented in Fig. 1(c). We notice that the formation energies are reduced substantially compared to $\mathrm{N}$-free cases. This is very exceptional, the mechanism being valid only for Ga interstitials inside a Ga tetrahedron. Nitrogen atoms stabilize the $\mathrm{Ga}$ interstitial atom in the model structure by pulling out the $\mathrm{Ga}$ atoms in the tetrahedron surrounding the interstitial $\mathrm{Ga}$ atom. This is most clearly verified by considering a situation where the $\mathrm{N}$ atoms are not nearest neighbors of the Ga tetrahedron atoms, and therefore do not pull them [Fig. 2(b)]. In this case, the formation energy for the neutral $\mathrm{Ga}$ interstitial atom is about $1.46 \mathrm{eV}$ larger. To test the mechanism for other dilute nitrides, we considered $\mathrm{GaP}$ and InAs, for which the corresponding energy differences are 1.43 and $1.70 \mathrm{eV}$, respectively. Note that GaP-based nitrides 


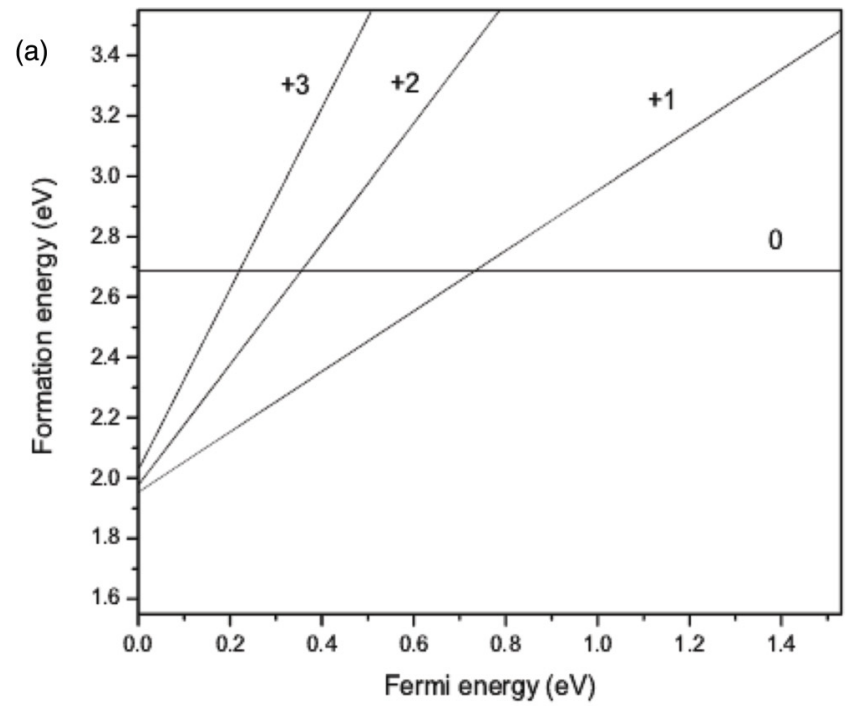

(a)

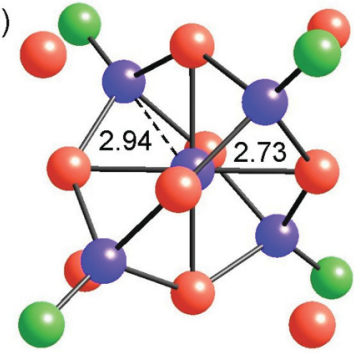

(b)

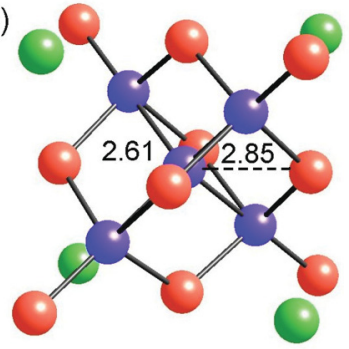

FIG. 2. (Color online) The energetically most favorable model structure for a $\mathrm{Ga}$ interstitial atom in $\mathrm{GaAsN}$ (a). Inequivalent structure for a $\mathrm{Ga}$ interstitial in GaAsN (b). Ga-As and $\mathrm{Ga}-\mathrm{Ga}$ distances in $\AA$ around the Ga interstitial are also shown. Ga, As, and $\mathrm{N}$ atoms are represented by blue, red, and green spheres, respectively.
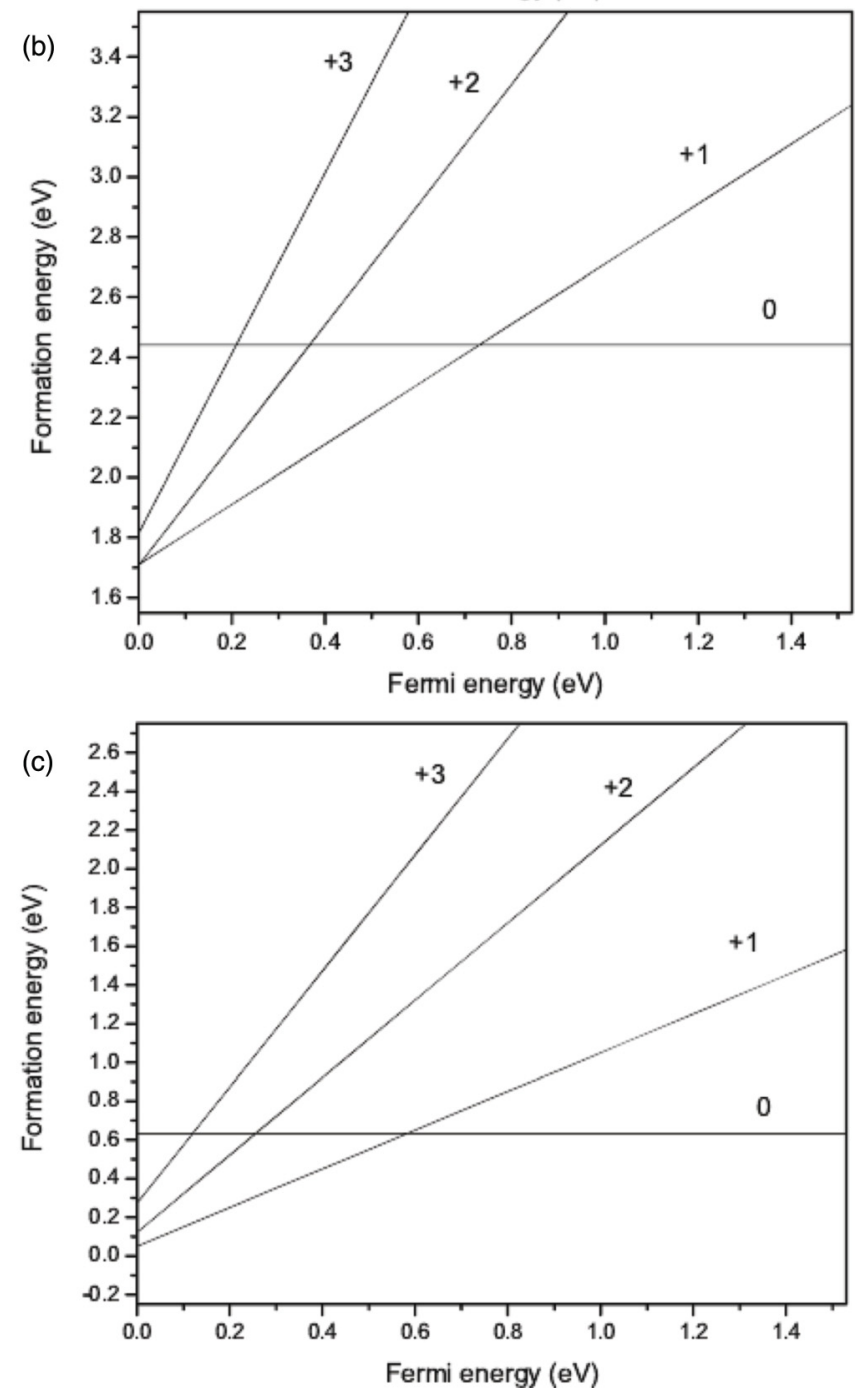

FIG. 1. Formation energies of Ga interstitial atoms in pure GaAs in the center of an As (a) and Ga (b) tetrahedron. Formation energy for a $\mathrm{Ga}$ interstitial atom in GaAsN within a model structure (c). The reference energy for the $\mathrm{N}$-doped $\mathrm{GaAs}$ without the interstitial atom is taken from the configuration in which the $\mathrm{N}$ atoms are maximally distant from each other. Formation energies are shown for different charge states. All figures represent Ga-rich conditions.

have also shown Ga interstitials. ${ }^{41,42}$ The Ga-Ga and Ga-As bond lengths around the interstitial are $2.94 \AA(2.61 \AA)$ and $2.73 \AA$ ( $2.85 \AA)$ respectively in the structure shown in Fig. 2(a) [Fig. 2(b)]. The same mechanism is not possible for the Ga interstitial atom inside an As tetrahedron. The interstitial $\mathrm{N}$ atom within the model structure is not stabilized either (the formation energy is very high, being $2.98 \mathrm{eV}$ in the neutral state). The $\mathrm{N}$ atoms restore a bulklike environment with nearest neighbor As atoms for the Ga interstitials in the model structure. Moreover, there is a larger charge density between the $\mathrm{Ga}$ and As atoms in the $\mathrm{N}$-induced model structure than between the $\mathrm{Ga}$ atoms in the nonreconstructed structure around the Ga interstitial.

It is interesting to note that the $\mathrm{N}$-stabilized interstitial $\mathrm{Ga}$ atom forms a nondispersive band within the bottom of the conduction band along the simple cubic $X-M$ symmetry direction (not shown). Band characters show that this band has a large contribution from the considered interstitial $\mathrm{Ga}$ atom. The density-of-states curve shows the feature within the bottom of the conduction band in Fig. 3 at $1.0 \mathrm{eV}$ above the top of the valence band.

Next, we considered the formation of the $\mathrm{N}$-induced $\mathrm{Ga}$ interstitial at the surface. To this end, we use a $(2 \times 1)$ structure within an $(8 \times 8)$ surface slab cell. The topmost $\mathrm{N}$ atoms within the model structure substitute the As dimer atoms. We found that in Ga-rich conditions the formation energy of the N-induced neutral Ga interstitial atom becomes approximately zero $(0.01 \mathrm{eV})$. Due to the slab construction used we could not consider charged interstitials, but it is assumed that, analogous to the bulk, the formation energies of the positively charged Ga interstitial atoms are lower than that of neutral interstitials, suggesting negative formation energies for positively charged Ga interstitials at surfaces. Wang et al. ${ }^{23}$ reported three different kinds of $\mathrm{N}$-induced $\mathrm{Ga}$ interstitial atoms under molecular beam epitaxial growth differing in electron localization. These can be explained if we assume that two, three, or four $\mathrm{N}$ atoms are needed in our model structure to stabilize these defects on a surface. The formation energy of the neutral Ga interstitial atom on the surface (in the bulk) with two $\mathrm{N}$ atoms is $0.52 \mathrm{eV}(1.70 \mathrm{eV})$. The formation energy for the interstitial with three $\mathrm{N}$ atoms is smaller (larger) than that of the interstitial with two (four) $\mathrm{N}$ atoms. Thus, all three kinds 


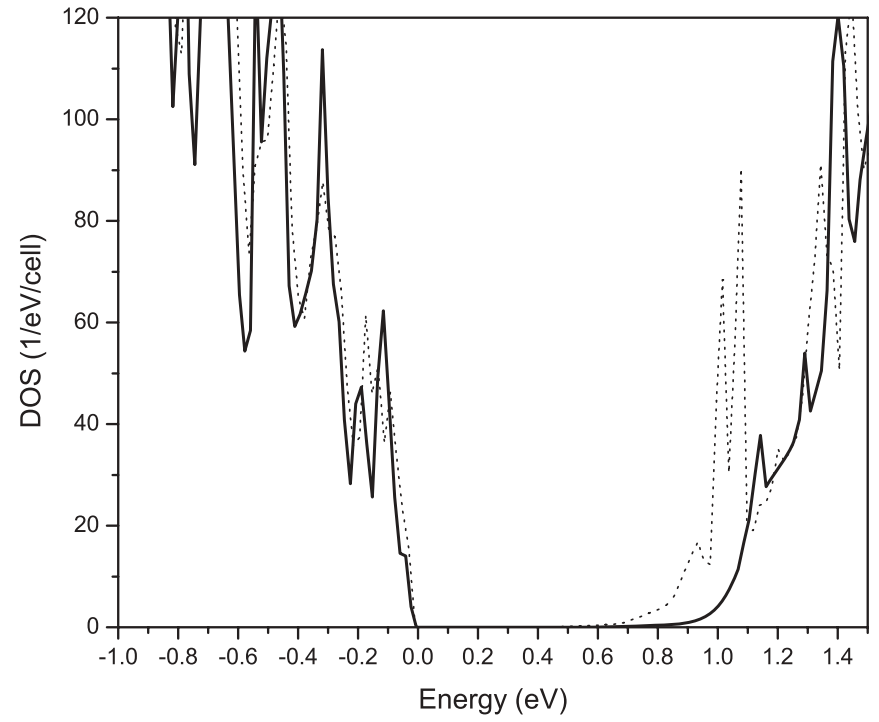

FIG. 3. Total density of states (DOS) for pure GaAs (solid line) and for GaAsN with the model structure shown in Fig. 2(a) buried in the GaAs (dotted line). The DOS curves have been aligned at the top of the valence band. The band induced by Ga interstitial within the model structure is shown about $1.0 \mathrm{eV}$ above the top of the valence band. The band gap is smaller than deduced from the figure, because the DOS at the bottom of the conduction band is very small. The magnitude of the band gap for GaAs agrees with that of Ref. 25 .

of interstitials may be formed during the crystal growth, while annealing will remove a large part of the less stable defects. ${ }^{23}$

We found that $\mathrm{N}$ atoms avoid nearest As substitutional sites. The next nearest sites are favored with respect to nearest substitutional sites by $0.13 \mathrm{eV}$ per $\mathrm{N}$ pair in the bulk, which may facilitate the formation of the $\mathrm{N}$-induced $\mathrm{Ga}$ interstitials. This is in agreement with the results presented in Ref. 43. On the other hand, the next nearest sites on the surface layer are favored by $0.32 \mathrm{eV}$ compared to the nearest sites.

We also consider two other representative and relatively stable defects, i.e., $\mathrm{N} t^{\mathrm{Ga}}$ interstitial and $(\mathrm{N}-\mathrm{N})_{\text {As }}$ split interstitial. ${ }^{25}$ We found that the $\mathrm{N} t{ }^{\mathrm{Ga}}$ defect is not even metastable in the neutral state on the surface, as the $\mathrm{N}$ atom moves to an As dimer site shifting the As dimer atom above the original dimer. The formation energy of the $(\mathrm{N}-\mathrm{N})_{\text {As }}$ split interstitial in the second As layer is $1.71 \mathrm{eV}$ larger than that of two $\mathrm{N}_{\mathrm{As}}$ atoms in the corresponding layer. Thus, surface effects do not favor the formation of the $\mathrm{N} t^{\mathrm{Ga}}$ interstitials and the $(\mathrm{N}-\mathrm{N})_{\mathrm{As}}$ split interstitials.

Experimental and the $a b$ initio core-level shifts (CLS) calculated for various models are shown in Table I. Intensities of different $\mathrm{N}$ components did not reveal any dependence on surface sensitivity which was found by varying the electron kinetic energy in the measurements [Figure 4(a)]. Therefore, it is clear that the $\mathrm{N}$ components presented below are derived from bulk.

Figure 4(a) shows that the $\mathrm{N} 1 \mathrm{~s}$ emission includes at least three components around $+0.2 \mathrm{eV}$ (C1), $0 \mathrm{eV}$ (C2), and $-0.4 \mathrm{eV}$ (C3). Table I shows that the $\mathrm{N}$ CLS of $\mathrm{N}_{\mathrm{As}}, \mathrm{N}_{\mathrm{As}}$ clusters, and $\mathrm{N}_{\mathrm{As}}$ clusters including $\mathrm{Ga}$ interstitials almost overlap. Therefore, most probably both $\mathrm{C} 1$ and $\mathrm{C} 2$ components represent these configurations possibly differing also with respect to final-state screening effects. The theoretical $\mathrm{N}$ shift of $-0.42 \mathrm{eV}$ matches the experimental component $\mathrm{C} 3$. However, the formation energy of $\mathrm{N} t^{\mathrm{As}}$ is very high $(4.11 \mathrm{eV}$ being the lower limit). Therefore, the $\mathrm{C} 3$ is not understood so far. The large-scale $\mathrm{N}$ spectra in the inset of Fig. 4(a) show that there are no larger $\mathrm{N} 1 s$ shifts compared to $\mathrm{C} 1-\mathrm{C} 3$.

It is remarkable that the experimental $\mathrm{N} 1 s$ CLS are much smaller than the theoretical ones (differences are significantly larger than the estimated maximum error for these theoretical bulk CLS, i.e. $0.1-0.2 \mathrm{eV}$ ) calculated for the following, previously considered $\mathrm{N}$ defects: the $\mathrm{N}-\mathrm{N}$ and $\mathrm{N}-\mathrm{As}$ split

TABLE I. Calculated initial-state core-level shifts (CLS) for different $\mathrm{N}$-induced defects. The minus sign denotes smaller binding energy compared to the reference, which is GaAs bulk emission for As and Ga levels and normal substitution, $\mathrm{N}_{\mathrm{As}}$, emission for N. Calculated shifts smaller than $0.05 \mathrm{eV}$ in absolute value are not listed. Results are for neutral defects. Clusters of two and four $\mathrm{N}_{\mathrm{As}}$ refer to one-dimensional and three-dimensional $\mathrm{N}_{\mathrm{As}}$ clusters, in which the separate $\mathrm{N}_{\mathrm{As}}$ atoms in one-dimensional pairs occupy next-nearest-neighbor As substitutional sites. Ga nn vacancy and Ga nn interstitial refer to construction in which the nearest-neighbor Ga or nearest Ga $t^{\mathrm{Ga}}$ interstitial site of an $\mathrm{N}_{\mathrm{As}}$ atom is filled with a vacancy or Ga atom defect, respectively. Measured CLS are also shown.

\begin{tabular}{llll}
\hline \hline & \multicolumn{1}{c}{ As } & Ga & $\mathrm{N}$ \\
\hline$(\mathrm{N}-\mathrm{N})_{\mathrm{As}}$ & -0.07 & $-0.05,+0.16$ & $-0.09,+1.48$ \\
$(\mathrm{~N}-\mathrm{As})_{\mathrm{As}}$ & $-0.12,+0.28$ & $-0.62,-0.07$ & -1.57 \\
$\mathrm{As}_{\mathrm{Ga}}-\mathrm{N}_{\mathrm{As}}$ & $-0.13,-0.05,+0.33$, & $-0.09,+0.06$ & -1.13 \\
$\mathrm{~N}_{\mathrm{Ga}}$ & +0.46 & $-0.61,-0.18,-0.07$ & +0.89 \\
$\mathrm{~N} t^{\mathrm{Ga}}$ & $-0.46,-0.13,-0.07$ & $-0.06,+0.07$ & -1.73 \\
$\mathrm{~N}-\mathrm{N} t^{\mathrm{Ga}}$ & -0.07 & $-0.13,-0.07$ & $+1.78,+2.04$ \\
$\mathrm{~N} t^{\mathrm{As}}$ & +0.14 & $-0.20,-0.14,+0.07$ & -0.42 \\
$\mathrm{~N}-\mathrm{N} t^{\mathrm{As}}$ & +0.09 & $-0.07,+0.26$ & $+1.54,+2.24$ \\
$\mathrm{Cluster}$ of two $\mathrm{N}_{\mathrm{As}}$ & & $-0.09,+0.25$ & +0.06 \\
$\mathrm{Cluster}$ of four $\mathrm{N}_{\mathrm{As}}$ & -0.05 & $-0.05,+0.10,+0.26,+0.49$ & -0.15 \\
Ga model interstitial & +0.20 & $-0.10,+0.18,+0.23,+0.34$ & -1.36 \\
Ga nn interstitial & +0.12 & $-0.28,-0.20,-0.10$ & $-0.4,0,+0.2$ \\
Ga nn vacancy & $-0.31,-0.26,-0.20,-0.11$ & $-0.16,+0.08,+0.25,+0.51$ & \\
Measured & $-0.31,+0.23,+0.63$ & & \\
\hline \hline
\end{tabular}




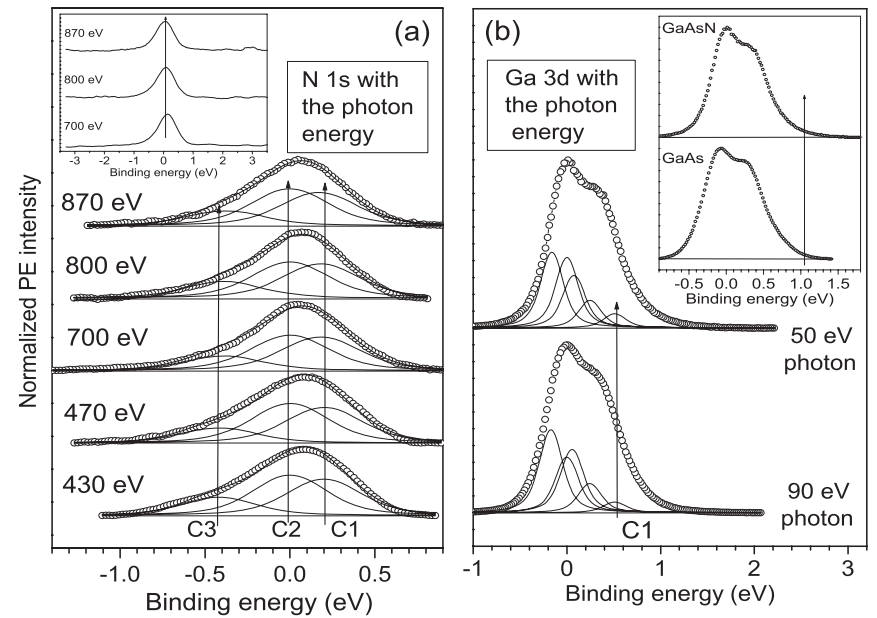

FIG. 4. (a) Photoemission N $1 s$ spectra from GaAsN measured at different surface sensitivity conditions by changing photon energy (i.e., electron kinetic energy). The $470 \mathrm{eV}$ spectrum is the most surface sensitive one, and bulk sensitivity increases toward the $870 \mathrm{eV}$ spectrum as well as toward the $430 \mathrm{eV}$ one. The inset shows large-scale spectrum. (b) Photoemission Ga $3 d$ spectra from GaAsN. The $90 \mathrm{eV}$ spectrum is more surface sensitive than the $50 \mathrm{eV}$ one. The inset shows comparison between $(2 \times 4)$ pure GaAs and GaAsN films.

interstitials, $\left(\mathrm{N}_{\mathrm{As}}-\mathrm{As}_{\mathrm{Ga}}\right)_{\mathrm{nn}}$, single $\mathrm{N} t^{\mathrm{Ga}}$ interstitial, as well as the $\mathrm{N}-\mathrm{N} t^{\mathrm{Ga}}$ and $t^{\mathrm{As}}$ interstitial defects.

The measured sample still included some extra As atoms on the surface after the decapping because the As $3 d$ emission included a contribution of additional As $(+0.63 \mathrm{eV})$, as identified previously. ${ }^{38}$ The Ga $3 d$ emission from GaAsN [Fig. 4(b)] reveals one characteristic component: $\mathrm{C} 1$ around $+0.5 \mathrm{eV}$, which has not been found for the pure $(2 \times 4)$ GaAs surface. ${ }^{38}$ This CLS may be attributed to Ga interstitial atoms. Note that only the $d_{5 / 2}$ peaks of the Ga $3 d$ double peak components are shown in Fig. 4(b) for clarity. The GaAsN-related component $\mathrm{C} 1$ appears as a high binding energy tail, as compared to the pure GaAs spectrum. This tail is marked by an arrow in the inset of Fig. 4(b), which also shows an approximated position of the $d_{3 / 2}$ peak of the $\mathrm{C} 1$ doublet. The intensity of this $\mathrm{C} 1$ component decreased for another GaAsN film with smaller $\mathrm{N}$ content of $2.6 \%$. On the other hand, the photoluminescence (PL) measurements showed that the PL intensity increased with decreasing the $\mathrm{N}$ content.

The PL spectra in Fig. 5 show a clear decrease in the PL intensity (i.e., increase in the defect amount) when the $\mathrm{N}$ concentration of GaAsN increases. In each comparison, the $\mathrm{N}$ plasma power is the same, because it is known that the $\mathrm{N}$ plasma power affects the PL intensity (i.e., intensities obtained by different plasma powers should not be compared). In contrast, the $\mathrm{N}$ flow is increased to increase the $\mathrm{N}$ content.

The $\mathrm{N}$ atoms in the model structure pull out $\mathrm{Ga}$ atoms around the interstitial $\mathrm{Ga}$, thus stabilizing the interstitial defect. Therefore, it is easy to think that substitutional atoms of large size could force the Ga neighbors of the interstitial remain closer to the positions in pure GaAs, thus destabilizing this defect. This is indeed the case if the $\mathrm{N}$ atoms in the model structure are substituted by $\mathrm{Bi}$ atoms. The formation energy of the $\mathrm{Ga}$ interstitial is increased by $2.60 \mathrm{eV}$, which means that in GaAsBi there should not exist Ga interstitial defects. The
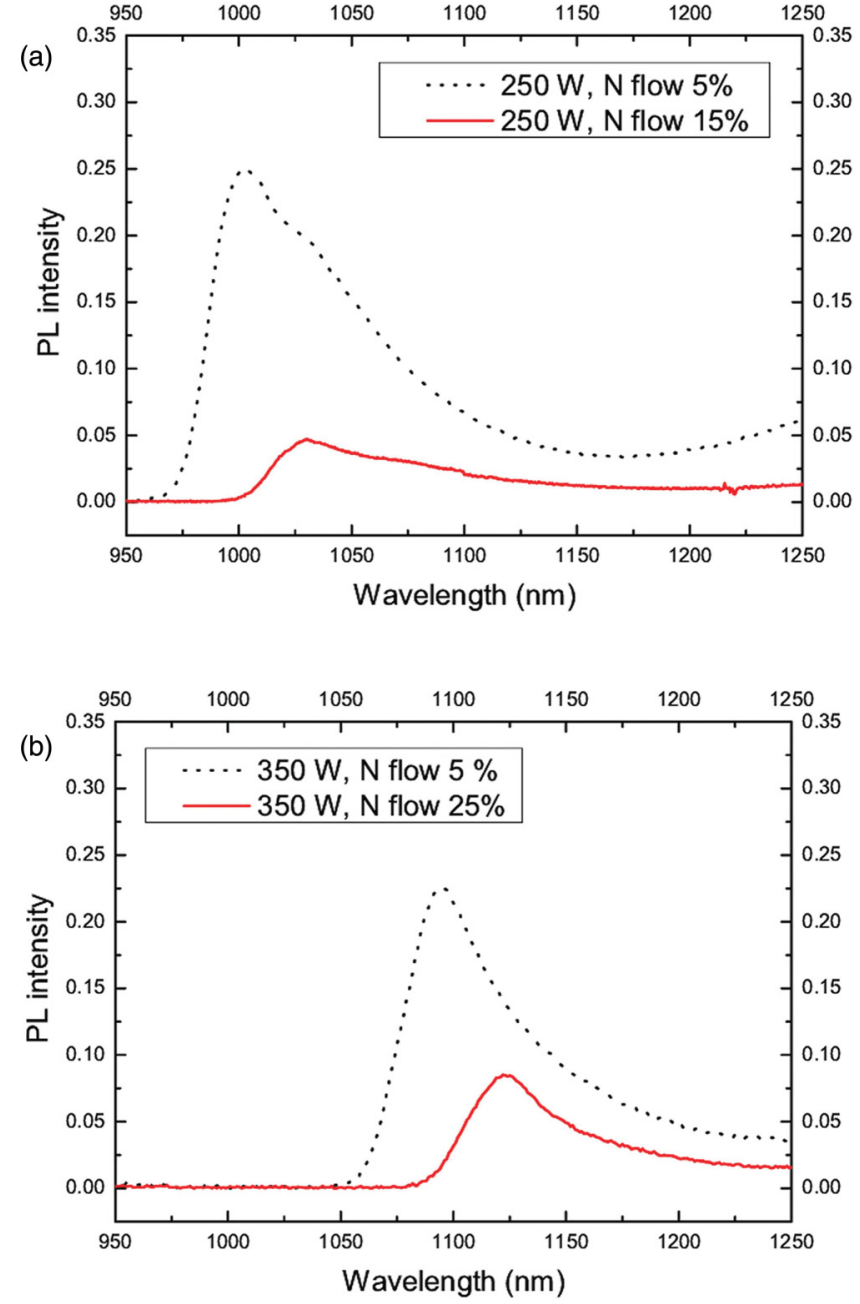

FIG. 5. (Color online) Photoluminescence spectra from GaAsN films grown with the same $\mathrm{N}$ plasma power but with different $\mathrm{N}$ flows. (a) N plasma power is $250 \mathrm{~W}$. X-ray diffraction analysis shows that $\mathrm{N}$ concentration is $1.17 \%$ with the smaller flow and $1.50 \%$ with the larger flow. (b) $\mathrm{N}$ plasma power is $350 \mathrm{~W}$. X-ray diffraction analysis shows that $\mathrm{N}$ concentration is $2.23 \%$ with the smaller flow and $2.53 \%$ with the larger flow.

$\mathrm{Ga}-\mathrm{Ga}$ and $\mathrm{Ga}-\mathrm{As}$ bond lengths around the interstitial are 2.56 and $2.88 \AA$ in this case. We should note that the use of $\mathrm{Bi}$ is very interesting also because it increases the maximum value of the valence band ${ }^{44}$ and provides stronger band bowing in combination with $\mathrm{N}$, finally enabling to us achieve band gaps smaller than $1 \mathrm{eV}$ while ensuring lattice matching to GaAs.

To avoid harmful Ga interstitials in GaAsN one may search within the Ga sublattice for additional dopants which would stick to $\mathrm{N}$ atoms and destabilize the $\mathrm{Ga}$ interstitials. Large atoms substituting the $\mathrm{Ga}$ neighbors of the $\mathrm{Ga}$ interstitial do not generally increase much the formation energy of the interstitials. This is understood, because although the large substituting atom would favor longer bonds with $\mathrm{N}$ than $\mathrm{Ga}$, thus approaching the interstitial atom; it also favors longer bonds with the neighboring As atoms, thus moving away from the interstitial.

Several additional dopants were considered from the III A, IV A, III B, and IV B groups of the periodic table (Y, La, Ti, Zr, 
Hf, Th, B, In, Tl, Si, Sn, and Pb). Results show that Zr, Hf, Th, and In increase the formation energy of the Ga interstitial atom significantly by $0.33,0.81,0.37$, and $0.35 \mathrm{eV}$, respectively, if these dopants are the nearest neighbors of the $\mathrm{Ga}$ interstitial atom (within the Ga sublattice). If the dopants are not nearest neighbors of the Ga interstitial atom (but still nearest neighbors of the $\mathrm{N}$ atoms), the corresponding values are $0.58,0.65,1.61$, and $0.00 \mathrm{eV}$. Other considered dopants do not increase the formation energy of the Ga interstitials significantly. Group IV $\mathrm{B}$ atoms also stick strongly to the $\mathrm{N}$ atoms. For example, the total energy of a pair of $\mathrm{Hf}$ (In) and $\mathrm{N}$ atoms in the GaAs unit cell is decreased by $1.10 \mathrm{eV}(0.22 \mathrm{eV})$ as the $\mathrm{Hf}(\mathrm{In})$ and $\mathrm{N}$ atoms become nearest neighbors, while the initial positions are maximally distant from each other. Therefore, some heavier IVB elements should destabilize the Ga interstitial atoms. Charge density analysis shows that the Hf atoms (group IV B atom) form stronger bonds with the neighboring As atoms than the Sn atoms (group IV A atom) do. On the other hand, the Sn atoms concentrate more electrons to the $\mathrm{N}$ atoms. However, the $\mathrm{Ga}$ interstitial atom weakens relatively much $\mathrm{Hf}-\mathrm{As}$ bonds (Hf-As bond length is increased about $0.1 \AA$ ), which leads to the destabilization of the $\mathrm{Ga}$ interstitial. The key difference with respect to the formation energy of the Ga interstitial between the IV A and IV $\mathrm{B}$ additional atoms may be in the electronegativities.

\section{CONCLUSIONS}

We have shown that $\mathrm{Ga}$ interstitials may be formed through $\mathrm{N}$-induced local effects (which restore a bulklike environment for the $\mathrm{Ga}$ interstitials) and surface effects. Nitrogen atoms avoid nearest As substitutional sites which may facilitate the formation of the environment favorable to the $\mathrm{Ga}$ interstitials. The $\mathrm{N}$-induced environment on the surface makes the formation energy approximately zero for a neutral defect. On the other hand, the formation energies of the competing $\mathrm{N}$-induced defects are not decreased significantly on the surface. These results suggest that the Ga interstitial atom is a much more abundant defect in GaAsN than the previously considered $\mathrm{N}$ defects, which have relatively large formation energies. Our photoemission measurements support these results. To counteract this, $\mathrm{Zr}$, $\mathrm{Hf}$, or Th atoms should be able to destabilize the harmful Ga interstitials.

\section{ACKNOWLEDGMENTS}

We are grateful to the MAX-lab staff and H. Ollila for their assistance and to T. Ahlgren for valuable discussions. The calculations were performed using the facilities of the Finnish IT Center for Science (CSC) and the Mgrid project (Turku, Finland). The Finnish Academy of Science and Letters, the National Graduate School in Materials Physics, Solar III-V TEKES project (Dnro: 3120/31/08), the Swedish Research Council, the European Research Council, the Hungarian Scientific Research Fund (research project OTKA 84078) and the Göran Gustafsson Foundation are also acknowledged for financial support. K.P. acknowledges support of the OTKA PD83353 project and the Bolyai Grant of the HAS.
*Corresponding author: marpunk@utu.fi.

${ }^{1}$ M. Kondow, K. Uomi, A. Niwa, T. Kitatani, S. Watahiki, and Y. Yazawa, Jpn. J. Appl. Phys. 35, 1273 (1996).

${ }^{2}$ Physics and Applications of Dilute Nitrides, edited by I. A. Buyanova and W. M. Chen (Taylor \& Francis, New York, 2004).

${ }^{3}$ Dilute Nitride Semiconductors, edited by M. Henini (Elsevier, Amsterdam, 2005).

${ }^{4}$ M. Guina, T. Leinonen, A. Härkönen, and M. Pessa, New. J. Phys. 11, 125019 (2009).

${ }^{5}$ R. Stevenson, Comp. Semicond. 17(5), 18 (2011).

${ }^{6}$ S.-H. Wei and A. Zunger, Phys. Rev. Lett. 76, 664 (1996).

${ }^{7}$ J. D. Perkins, A. Mascarenhas, Y. Zhang, J. F. Geisz, D. J. Friedman,

J. M. Olson and S. R. Kurtz, Phys. Rev. Lett. 82, 3312 (1999).

${ }^{8}$ A. Lindsay and E. P. O’Reilly, Phys. Rev. Lett. 93, 196402 (2004).

${ }^{9}$ D. Karaiskaj, A. Mascarenhas, M. Adamcyk, E. C. Young and T. Tiedje, Phys. Rev. B 74, 035208 (2006).

${ }^{10}$ M. Guina and S. H. Wang, in Molecular Beam Epitaxy, edited by M. Henini (Elsevier, Amsterdam, in press).

${ }^{11}$ H. P. Xin and C. W. Tu, Appl. Phys. Lett. 72, 2442 (1998).

${ }^{12}$ W. Li, J. Turpeinen, P. Melanen, P. Savolainen, P. Uusimaa, and M. Pessa, Appl. Phys. Lett. 78, 91 (2001).

${ }^{13}$ S. G. Spruytte, C. W. Coldren, J. S. Harris, W. Wampler, P. Krispin, K. Ploog, and M. C. Larson, J. Appl. Phys. 89, 4401 (2001).

${ }^{14}$ S. B. Zhang and S.-H. Wei, Phys. Rev. Lett. 86, 1789 (2001).
${ }^{15}$ K. M. Yu, W. Walukiewicz, J. Wu, D. E. Mars, D. R. Chamberlin, M. A. Scarpulla, O. D. Dubon, and J. F. Geisz, Nat. Mater. 1, 185 (2002).

${ }^{16}$ A. Janotti, S.-H. Wei, S. B. Zhang, S. Kurtz, and C. G. Van de Walle, Phys. Rev. B 67, 161201(R) (2003).

${ }^{17}$ P. Carrier, S.-H. Wei, S. B. Zhang, and S. Kurtz, Phys. Rev. B 71, 165212 (2005).

${ }^{18}$ E. Arola, J. Ojanen, H.-P. Komsa, and T. T. Rantala, Phys. Rev. B 72, 045222 (2005).

${ }^{19}$ T. Ahlgren, E. Vainonen-Ahlgren, J. Likonen, W. Li, and M. Pessa, Appl. Phys. Lett. 80, 2314 (2002).

${ }^{20}$ M. Reason, H. A. McKay, W. Ye, S. Hansin, R. S. Goldman, and V. Rotberg, Appl. Phys. Lett. 85, 1692 (2004).

${ }^{21}$ P. Krispin, V. Gambin, J. S. Harris, and K. H. Ploog, J. Appl. Phys. 93, 6095 (2003).

${ }^{22}$ X. J. Wang, I. A. Buyanova, F. Zhao, D. Lagarde, A. Balocchi, X. Marie, C. W. Tu, J. C. Harmand, and W. M. Chen, Nat. Mater. 8, 198 (2009).

${ }^{23}$ X. J. Wang, Y. Puttisong, C. W. Tu, A. J. Ptak, V. K. Kalevich, Yu. Egorov, L. Geelhar, H. Riechert, W. M. Chen, and I. A. Buyanova, Appl. Phys. Lett. 95, 241904 (2009).

${ }^{24}$ S. G. Spruytte, M. C. Larson, W. Wampler, C. W. Coldren, H. E. Petersen, and J. S. Harris, J. Cryst. Growth 227-228, 506 (2001).

${ }^{25}$ K. Laaksonen, H.-P. Komsa, T. T. Rantala, and R. M. Nieminen, J. Phys.: Condens. Matter 20, 235231 (2008). 
${ }^{26}$ D. M. Ceperley and B. J. Alder, Phys. Rev. Lett. 45, 566 (1980).

${ }^{27}$ J. P. Perdew and A. Zunger, Phys. Rev. B 23, 5048 (1981).

${ }^{28}$ P. E. Blöchl, Phys. Rev. B 50, 17953 (1994).

${ }^{29}$ G. Kresse and D. Joubert, Phys. Rev. B 59, 1758 (1999).

${ }^{30}$ G. Kresse and J. Hafner, Phys. Rev. B 47, 558 (1993).

${ }^{31}$ G. Kresse and J. Hafner, Phys. Rev. B 49, 14251 (1994).

${ }^{32}$ G. Kresse and J. Furthmüller, Comput. Mater. Sci. 6, 15 (1996).

${ }^{33}$ G. Kresse and J. Furthmüller, Phys. Rev. B 54, 11169 (1996).

${ }^{34}$ H. J. Monkhorst and J. D. Pack, Phys. Rev. B 13, 5188 (1976).

${ }^{35}$ M. Bernasconi, G. L. Chiarotti, and E. Tosatti, Phys. Rev. B 52, 9988 (1995).

${ }^{36}$ P. Spiewak, K. Sucoka, J. Vanhellemont, K. J. Kurzydlowski, K. Mlynarczyk, P. Wabinski, and I. Romandik, Physica B 401-402, 205 (2007).

${ }^{37}$ I. Vurgaftman, J. R. Meyer, and L. R. Ram-Mohan, J. Appl. Phys. 89, 5815 (2001).

${ }^{38}$ M. P. J. Punkkinen, P. Laukkanen, K. Kokko, M. Ropo, M. AholaTuomi, I. J. Väyrynen, H.-P. Komsa, T. T. Rantala, M. Pessa,
M. Kuzmin, L. Vitos, J. Kollár, and B. Johansson, Phys. Rev. B 76, 115334 (2007).

${ }^{39}$ S. B. Zhang and J. E. Northrup, Phys. Rev. Lett. 67, 2339 (1991).

${ }^{40}$ M.-A. Malouin, F. El-Mellouhi, and N. Mousseau, Phys. Rev. B 76, 045211 (2007).

${ }^{41}$ N. Q. Thinh, I. P. Vorona, I. A. Buyanova, W. M. Chen, S. Limpijumnong, S. B. Zhang, Y. G. Hong, H. P. Xin, C. W. Tu, A. Utsumi, Y. Furukawa, S. Moon, A. Wakahara, and H. Yonezu, Phys. Rev. B 71, 125209 (2005).

${ }^{42}$ I. P. Vorona, T. Mchedlidze, M. Izadifard, I. A. Buyanova, W. M. Chen, Y. G. Hong, H. P. Xin, and C. W. Tu, Appl. Phys. Lett. 86, 222110 (2005).

${ }^{43}$ A. Carvalho, S. J. Barker, R. Jones, R. S. Williams, M. J. Ashwin, R. C. Newman, P. N. Stavrinou, G. Parry, T. S. Jones, S. Öberg, and P. R. Briddon, Physica B 401-402, 339 (2007).

${ }^{44}$ Y. Zhang, A. Mascarenhas, and L.-W. Wang, Phys. Rev. B 71, 155201 (2005). 